\title{
Troubles dans la sexualité : «entre-deux » féminin et processus d'asexuation dans Les chambres de bois
}

\author{
LOIC BOURDEAU \\ UNIVERSITY OF LOUISIANA, LAFAYETTE
}

Résumé : Le présent article souhaite montrer comment le roman Les chambres de bois est construit sur une constante instabilité identitaire résultant en une asexualité. II s'agit de considérer Catherine comme étant en proie aux autres et non libre de s'affirmer. Ce faisant, elle oscille entre divers statuts (fille, mère, objet) qui l'éloignent en permanence de la sexualité. Qu'elle soit femme-mère, femme-objet ou femme-enfant, l'accès au désir n'est jamais vraiment autorisé et complet.

Mots-clés : Féminin, Les chambres de bois, Sexualité, Infantilisation, Objectification, Identité.

Anita se détendit, articula très doucement, comme à regret :

- Il faut que tu te maries, Catherine...

La jeune fille avala sa salive comme si elle étouffait :

- Avec Michel ?

- Avec Michel ? Bien sûr, avec qui veux-tu donc que ce soit ?

$[\ldots]$

- Tante Anita, j'ai peur que tout cela ne soit un rêve et que Michel ne m'épouse jamais. (Les chambres de bois, 1958 : 48-49)

L'univers des Chambres de bois est indiscutablement un univers à forte présence féminine ${ }^{1}$; une présence oppressée par le joug d'un patriarcat tout puissant. Le pas-

1. On peut de se demander si cette prédominance reflète un parti pris ou un simple choix stylistique et esthétique. Selon I'Histoire de la littérature québécoise - qui se penche notamment sur Gabrielle Roy, Rina Lasnier et Anne Hébert - « la littérature apparaît chez elles comme un engagement individuel et acquiert une autonomie qu'elle n'avait pas auparavant. Celle-ci semble aller de soi et rejoint un mouvement plus général qui vise à dégager la littérature locale des obligations politiques immédiate ». (Biron, 2010 : 281) Le présent roman reste, néanmoins, ancré dans un certain engagement à l'égard de la condition de la femme, ce que la référence à Green (2001 : 153) et cette analyse souhaitent mettre en avant. 
sage cité démontre notamment l'importance des traditions et combien elles sont ancrées dans les comportements de chaque personnage. Les femmes, surtout, sont dirigées par un désir de mariage présenté comme une étape inévitable, voire salvatrice, dans la vie d'une jeune fille. Pour Catherine qui vit dans un univers austère, de «brouillard » $\left(C B^{2}: 28\right)$, où dominent « de noirs palais d'Apocalypse » ( $\left.C B: 27\right)$, et qui passe son temps à « nourrir, laver, peigner, habiller et repriser [les trois petites sœurs], tandis que le père se retirait en sa solitude » $(C B: 27)$, le mariage - de surcroît avec Michel qui vivait dans «la maison des seigneurs » ( $C B: 33)$ - semble une échappatoire quasi-fantastique. De fait, la «peur» que ressent Catherine face à un potentiel rejet pourrait être, à première vue, la simple expression de sentiments amoureux et un réel désir de s'unir au jeune homme. Toutefois, cette peur - remise dans le contexte de l'époque - peut exprimer aussi le besoin de conformation aux valeurs traditionnelles et l'angoisse de ne pas plaire et de perdre un prétendant. Bien que Catherine envisage cette union comme un moyen de s'éloigner de son environnement originel, de noirceur et d'ennui, le roman s'attachera, en effet, à questionner l'influence sociale sur le comportement des individus, de même que l'agentivité de I'héroïne ${ }^{3}$. Bien évidemment, la thématique du féminin n'est en rien une observation nouvelle, dans ce qu'il s'agit d'un point d'intérêt prédominant pour les critiques de l'œuvre ${ }^{4}$. De plus, le féminin comme topos central du roman ne se limite pas à I'œuvre d'Anne Hébert. À juste titre, Green remarque que « writers like Noël, Théoret, Ouellette-Michalska, and Anne Hébert [...] share the project of giving voice to silenced women ». (Green, 2001 : 153)5 Néanmoins, cette analyse se propose de considérer spécifiquement deux problématiques en lien avec l'écriture du féminin dans Les chambres de bois. Tout d'abord, il convient de s'interroger sur les diverses stratégies qui enferment Catherine dans un état transitionnel, une constante instabilité de son statut que nous nommerons « entre-deux féminin ${ }^{6}$, et qui participe, de manière 2. CB pour Les chambres de bois, 1958.

3. Comprenons par ce terme les capacités décisionnelles d'un individu, de même que la faculté à agir, voire à influencer d'autres individus.

4. Comme nous pouvons le voir dans la bibliographie disponible sur le site du Centre Anne-Hébert, le travail de cette écrivaine a été analysé en profondeur. Au sujet du thème de la femme, outre les textes cités dans cette contribution, sont notables : «Amors i identidad a l'obra d'Anne Hébert, Les chambres de bois 》 (Anoll, 1996), «Catherine : Les chambres de bois 》 (Lapierre, 1998), «Au pays de la fille maigre : Les chambres de bois d'Anne Hébert » (Aylwin, 1967), ou encore « Autonomie et ordre maternel dans Les chambres de bois d'Anne Hébert » (Boisclair, 2005).

5. Notre traduction : « des écrivains comme Noël, Théoret, Ouellette-Michalska, et Anne Hébert [...] partagent le même dessein de rendre la parole à des femmes réduites au silence ».

6. Cette terminologie fait référence à un passage entre l'enfance et l'âge adulte, sans directement signifier I'adolescence. À vrai dire, I'emploi du mot « adolescence » conduit à des conceptions trop réductrices de la situation de Catherine. A contrario, « entre-deux féminin » permet une définition plus souple qui souligne la complexité de l'état dans lequel le personnage se trouve. Elle est enfant, adolescente, jeune fille à marier, mère-substitut. Elle est tout à la fois, elle se retrouve dans un état, 
inhérente, à l'impossibilité d'une réelle jouissance sexuelle et physique. Cette notion d'entre-deux a plusieurs buts. Dans un premier temps, elle viendra souligner la déshumanisation de I'héroïne (femme ou objet). Plus précisément, Hébert a recours à I'objectification et à la sacralisation qui sert à détacher Catherine du royaume du décisionnel, de l'actif, et anéantit toute possibilité de pénétrer le royaume du désir charnel. Plus tard aussi, nous verrons comment à travers un processus de « maternalisation » (dans le sens de « faire mère »), Catherine est condamnée à l'asexualité. À travers ces axes de recherche, nous soulignerons donc l'instabilité identitaire qui exclut la possibilité pour l'héroïne d'accéder à une sexualité épanouissante. Dans le même temps, l'on doit constater que certaines instances peuvent renforcer une conception sociétale normative quant à ce qui est acceptable ou non. Par exemple, les moments de transgression ayant principalement trait à l'inceste ou à la pédophilie sont révélés sous une lumière négative, résultant parfois de l'émergence d'un certain malaise chez le lecteur. Cependant, cela ne signifie en rien que l'auteure souscrive à de telles conceptions. Plutôt, ce malaise consolide la dimension non-jouissive, non-définie, et non-conforme de la vie de Catherine. En ce sens, ce n'est pas tant l'aspect transgressif qui met fin au plaisir lors des rapports entre Catherine et Michel, mais plutôt le fait que I'héroïne ne soit pas femme en pleine possession d'un soi stable et agentique.

Comme point de départ, il convient de considérer la question de l'âge et de la maturité. La thématique de l'enfance ou de l'émancipation chez Hébert a déjà fait l'objet - et à juste titre - de nombreuses recherches critiques (Bishop, 1977; Boisclair, $2000,2005,2008)$, que cette contribution prend comme point de départ avant d'en proposer une nouvelle lecture. Au-delà du fait que « la structure tripartite du roman Les chambres de bois figurait le passage de la femme dans l'économie patriarcale, jusqu'à son émancipation »(Boisclair, 2005 : 81), cette analyse souhaite rendre compte de l'état transitionnel et oscillatoire mentionné (et son lien à la sexualité). Si l'argument de Boisclair sur le rôle de la mère-in absentia est tout à fait convaincant, il semble également nécessaire de considérer en quoi l'émancipation n'est pas nécessairement un signe de maturité. Tout au long du récit, Catherine est en proie à une crise identitaire qui la fait osciller entre fille, mère, femme, et même objet. Cette analyse se concentre plus particulièrement sur ces moments de va-et-vient entre les différents statuts de I'héroïne, ainsi que sur les paradoxes et les contradictions qui participent de l'asexuation du personnage principal. À travers les trois catégories

puis dans un autre. Elle fait face à de constants remous identitaires, qui la placent dans un interstice instable du « soi ». 
principales qui émergent (fille, mère, femme), nous verrons que lorsque I'héroïne accède à un statut de sujet, elle reste toutefois dépendante de l'autre. Dans le même temps, I'infantilisation de l'héroïne et l'instabilité constante de son statut soulignent ces troubles et ces difficultés à se libérer.

\section{Entre instabilité et identité assignée}

Dès le début, « [I]e père au travail, les sœurs à l'école » ( $C B: 33)$, I'héroïne est au milieu, ni enfant, ni mère, « une grosse natte de cheveux battant dans son dos. » $(C B: 41)$ Ainsi, elle incarne, au sens le plus corporel qui soit, l'ambiguïté à son paroxysme. Que «l'âge de Catherine fût aussi peu sûr aux hanches et aux seins » $(C B$ : 37) n'est qu'une première représentation de cet entre-deux féminin qui la condamne à une certaine violence et la prédestine à être constamment en proie à l'autre?. Pour revenir à la citation en incipit, la volonté de la tante, Anita, de marier sa nièce, et le climat sinistre qui entoure les personnages, ne font qu'accentuer l'instabilité de Catherine. Est-elle vraiment sûre de vouloir se marier ? Il apparaît que l'union matrimoniale n'est qu'un acte de conformation aux lois sociétales, lui permettant ainsi d'échapper à l'atmosphère funeste de la maison d'enfance, tout en évitant la marginalisation. Nous avons alors affaire à une performance normative, dans ce que I'héroïne tente, sans réel succès, de suivre le chemin tracé par tant d'autres femmes avant elle. Dans cet environnement patriarcal, et surtout pour le mari, la femme constitue, dans une certaine mesure, une toile blanche sur laquelle s'impriment les volontés et désirs des autres ${ }^{8}$. De fait, malgré des moments de résistance, et la conscience de sa soumission, une fois mariée, elle « $\mathrm{s}^{\prime a p p l i q u a i t ~ a ̀ ~ d e v e n i r ~ c e ~}$ que Michel désirait qu'elle fût. » (CB:84) L'emploi du verbe « s'appliquer » (et plus loin «apprendre ») souligne de surcroît la dimension performative de ses actes. À ce propos et en relation avec le corps, Butler remarque que « to be a body is to be given over to others even as a body is, emphatically, "one's own" ». (Butter, $2004: 20)^{9}$ Le

7. Il me semble utile d'insister ici sur la souffrance que subit Catherine en relation avec l'instabilité identitaire. Bien que la femme, toute femme, devienne un objet sous I'ordre patriarcal, le fait que I'héroïne ait également besoin de découvrir son soi intérieur exacerbe cette souffrance. À devoir se conformer aux attentes de l'autre, à se mouler en fonction de désirs extérieurs, elle retarde le processus d'affirmation de son identité. Aussi, quoique Boisclair explique que « [I]a mère [a] réussi à arracher sa fille de l'ordre patriarcal et [s'est] assurée que celle-ci réalise une alliance amoureuse placée hors du signe de la servitude » (Boisclair, 2005 : 95), le roman maintient la jeune fille dans I'incertitude de savoir vraiment qui elle est. Une référence à « Was ist Aufklärung 》 (Kant, 1784) nous permettrait de voir que la fin de l'émancipation n'est que partielle et n'implique justement pas un réel «Aufklärung / éclairement ».

8. Bien consciente de son statut, Catherine remarque : « Je lui suis soumise, mais faites, ô mon dieu, qu'il me prenne sans me faire de mal ! » $(C B: 71)$

9. Notre traduction : « être un corps, c'est être donné à l'autre quand bien même ce corps est, clairement, "le nôtre" ». 
message a une portée d'autant plus frappante dans le cas de Catherine puisqu'étant sujette à l'instabilité elle ne peut qu'agir en fonction de ce que les autres décident. Elle subit leurs attaques tandis qu'ils tentent de la mouler selon leurs propres souhaits. Ainsi, si pour Butler nous sommes tous victimes de ce processus, nous sommes tous analysés et définis par les autres, cela n'entraîne pas forcément un changement de comportement. Dans le cas de Catherine, quand bien même elle souhaite changer, ses demandes ne font face qu'à des refus :

- Michel, je t'en prie, laisse-moi faire autre chose ! Le marché, les repas... oui, c'est cela ! J'aimerais faire un grand gâteau pour la Fête des Rois !

- Tu mêles tout, Catherine. [...] Je n'ai pas épousé une cuisinière, je pense ? $(C B: 83)$

L'on s'interroge alors : qui ou qu'a-t-il épousé ? De nouveau, la tentative de rejet d'un statut assigné par l'autre échoue et renforce le trouble quant à son identité, voire son utilité.

\section{Entre objectification et sacrifice}

Le participe passé « given » (donné) employé par Butler est encore plus pertinent pour Les chambres de bois, puisque Catherine ne fait que passer d'une identité à une autre. Il est alors peu surprenant que le champ lexical du don (ou du sacrifice) soit très présent à travers le récit, tel que Catherine « devenait pareille à une jeune offrande sur la table de pierre » $(C B: 71)$; elle « [avait] l'air d'une idole » ( $C B: 92)$, ce qui amplifie à la fois son impossibilité d'échapper à l'assignation de l'autre et son statut de proie. L'héroïne prend ici une dimension nouvelle qui éloigne plus encore l'acte sexuel. Tout d'abord, en étant assigné par Michel à un statut d'objet, elle ne peut pas ou plus être la cible d'une attraction physique et passionnelle. Mais surtout, en atteignant un rôle d'objet sacrificiel - un objet de don qui circule entre les hommes - elle prend une dimension quasi-sacrée et ne peut être souillée. Les passages suivants servent à faire la lumière sur cette transformation de l'héroïne :

J'irai te demander à ton père, s'il le faut. ( $C B: 64$; nous soulignons)

Il y eut d'abord tout un jour dans l'appartement de Michel, [...] entre les malles, les caisses, la poussière, le désordre figé de l'année dernière, [...] les sandwiches séchés, le feu qui ne prend pas [...]. Catherine se coucha dans le lit de Michel. (CB : 67; nous soulignons)

Ne m'abandonnez pas, Catherine, je n'ai plus que vous à présent... Il parlait comme s'il avait eu la fièvre, serrant les poignets de l'adolescente dans ses doigts. ( $C B: 63$; nous soulignons) 
Ces trois exemples insistent sur la dimension possessive du roman, à savoir que Catherine est clairement un objet. Nous avons affaire à l'emploi de compléments du nom (« de Michel », « de l'adolescente ») qui opèrent selon un mode de distanciation, c'est-à-dire que l'agentivité de Catherine est anéantie par le lien à un homme oppresseur. Une fois dans l'appartement, I'héroïne semble faire partie du décor, un objet de plus accumulé par Michel. L'expression «s'il le faut » $(C B: 64)$ est aussi un moyen de mettre en exergue le manque d'intérêt de Michel pour la fille. Dans cette conjoncture où la passion et le désir sont abandonnés au profit d'un besoin égoïste de ne pas être seul, il est dès lors évident que Catherine n'entre pas dans une union hommefemme pleine et physique. Catherine est ainsi la représentation ultime du don au profit de la continuation du patriarcat. Nancy Jay explique à propos des origines et du fonctionnement des rites de sacrifice que « $[\mathrm{w}]$ ithout marriage there can be no paternal filiation, no male line of descent, no family, all of which presuppose a link which is not natural, but religious and social. » (Vernant dans Jay, 1992 : 30) ${ }^{10}$ Bien que Michel ne soit pas un modèle traditionnel de masculinité - selon les prescriptions patriarcales et les attentes face à son genre -, il essaie aussi de se conformer aux attentes sociales et considère le mariage comme un moyen d'affirmer son statut d'homme et de continuer à faire vivre sa lignée et son héritage. Catherine représente l'objet pour parvenir à cette fin, mais il est trop hanté par le passé pour mener son projet à terme. À cela s'ajoute la dimension sacrée que prend Catherine. En effet, et pour comprendre encore mieux l'impact sur la sexualité, nous souhaitons parler de Catherine, la femme-objet en tant que femme-totem, ce qui exacerbe l'impossibilité du contact physique jouissif. Freud explique dans Totem et tabou :

Qu'est-ce qu'un totem? D'une façon générale, c'est un animal, comestible,
inoffensif ou dangereux et redouté [...]. Le totem est, en premier lieu, I'an-
cêtre du groupe; en deuxième lieu, son esprit protecteur et son bienfaiteur
qui envoie des oracles et, alors même qu'il est dangereux pour d'autres,
connaît et épargne ses enfants. Ceux qui ont le même totem sont donc
soumis à l'obligation sacrée, dont la violation entraîne un châtiment auto-
matique, de ne pas tuer (ou détruire) leur totem, de s'abstenir de manger
de sa chair ou d'en jouir autrement. (Freud, $1912: 7$; nous soulignons)

10. Notre traduction : «sans le mariage, il ne peut y avoir aucune filiation paternelle, aucune descendance de la lignée mâle, aucune famille, le tout présupposant ainsi un lien qui n'est pas naturel, mais religieux et social. » 
Nous avons vu plus haut le thème du sacrifice, et cette intervention freudienne confirme que le sacré sert à positionner Catherine comme être-objet et femme-protectrice (de Michel) ${ }^{11}$. Si le mari vénère la femme, ce n'est qu'à des fins narcissiques: elle n'est qu'une « offrande » pour garantir son bien-être. En résulte aussi, de façon inévitable, un écartement du corps, une interdiction face à la chair, dont il ne faut pas jouir. Par peur de la violation (dont nous verrons les conséquences ci-dessous), Michel s'arrange pour que son épouse ne soit plus qu'une image (I'idole) intouchable, non-désirable ${ }^{12}$. Quand bien même, à la fin de la première partie, Michel la caresse « comme s'[il] eût voulu susciter, sans hâte ni passion, un corps solide et doux » ( $C B: 64$ ), le manque de désir traduit d'abord un certain mécanisme automatique. Il tente d'agir en homme, en mari, mais n'y parvient pas. Le plaisir est donc principalement gâché dans la mesure où il est constamment conscient de la violation dont il est l'auteur, et ne peut s'empêcher de ne percevoir Catherine que comme un substitut ${ }^{13}$.

11. S'il peut paraître surprenant d'avoir recours à Freud pour traiter de sujets féministes et de la sexualité des femmes, il est nécessaire de garder en tête qu'a contrario des autres médecins de l'époque qui enfermaient les femmes « malades», Freud les analysait et en formait même à la psychanalyse. Un très grand nombre de contributions académiques confirment également que Freud et le féminisme ne sont point incompatibles. Entre autres, voir Juliet Mitchell, Psychoanalysis and Feminism : A Radical Reassessment of Freudian Psychoanalysis (1974); mais aussi les travaux de Mélanie Klein, ou de Nancy Chodorow. De même, il n'apparaît pas contradictoire de convoquer Butler et Freud conjointement, en ce sens que Butler réfléchit à des notions issues de ce penseur. Voir, notamment, The Psychic Life of Power: Theories in Subjection (1997).

12. En relation avec l'idée d'une femme-image/femme-objet, nous attirons l'attention sur Russell pour qui : « He [Michel] is attracted to the perfection represented by a complete absence of life, a complete absence of color. » (Russel, 1983 : 53) Cet attrait pour l'absence de couleur fait écho au passage suivant : « Je veux te peindre en camaïeu, toute blanche, sans odeur, fade et fraîche comme la neige, tranquille comme l'eau dans un verre. » (CB:83) Cette palette de couleur pâle et naturelle soutient alors I'hypothèse que Catherine fait, en quelque sorte, partie du paysage. Elle se fond dans le décor. Sans passion (pas de couleur chaude), elle est la perfection, l'image désirée qu'on ne peut approcher. Notons également que «Catherine prenait place [sur une haute chaise], rangée par la servante » $(C B: 87)$, tel un bibelot que I'on tient à distance.

13. Émond note de façon très claire que Michel «craint par-dessus tout les feux de la passion » (Émond, $1984: 191$ ), ce qui contraste dramatiquement avec l'atmosphère environnante : « Les hauts fourneaux des Chambres de bois ressemblent à une fournaise infernale à laquelle seraient condamnés les hommes ». (Émond, $1984: 189$ ) Nous irons plus loin en disant que les hauts fourneaux sont un important symbole phallique qui, en quelque sorte, marque la prédominance sociale du masculin et projette sur Michel une norme de masculinité qu'il ne peut atteindre. Il est alors condamné à ne pouvoir assurer son rôle. 


\section{Entre agentivité et maternité}

Lorsque Catherine parvient à atteindre un certain statut de sujet ${ }^{14}$, quoique toujours dépendante de la volonté de Michel, elle abandonne un état d'objectification pour se substituer à un rôle maternel. Dans ce sens, notons les commentaires suivants : «Catherine comprend à la rencontre à l'extérieur de la maison (car elle n'y entre jamais) que Michel ne la veut que comme substitut de Lia ». (Carlshamre, 2009 : 67) La thématique de la substitution est également mise en exergue, lorsque nous lisons que «Catherine et Lia, dans Les chambres de bois, sont la doublure I'une de l'autre ». (Bouchard, 1977 : 14) Or, il apparaît que Catherine est bien plus qu'un substitut sororal, mais avant tout un substitut de la mère. Elle sert de lien maternel en l'absence de la sœur, elle réconforte et se met à agir comme une mère lorsque frère et sœur sont réunis. Pour mieux comprendre ce concept, il est nécessaire de considérer l'infantilisation de Michel (et de Lia). La peur de l'abandon renvoie à la perte de la mère, comme le rappelle ce souvenir : «La mère est partie depuis cinq mois, déjà. Le père chasse tout le jour [...] et les deux enfants, seuls, [...] font un pacte et se jurent fidélité ! » ( $C B: 127)$ Ainsi, quand Lia quitte son frère pour un homme, elle répète cet épisode passé, brise leur pacte et plonge Michel dans une grande détresse. L'union à Catherine prend tout son sens dans ce qu'elle devait assurer à Michel une stabilité maternelle, c'est-à-dire que le lien officiel et civil (du mariage) prolonge une certaine dépendance à laquelle Lia, en tant que sœur, n'a pas à souscrire. Dans le même temps, cela met en avant le fait que Michel est resté un enfant. Quand, un soir, il « entoura de ses bras les jambes de Catherine, enfouit sa tête dans les plis de sa robe et flaira la baie chaude des genoux $\gg(C B: 82)^{15}$, il y a comme un désir de régression in utéro. Le mariage a donc permis de récréer un cordon ombilical qui ne suffit pas à calmer les angoisses; et la femme-objet est alors remplacée par une femme-mère. L'échange entre Michel et Lia vient confirmer la mort de Catherine en tant que femme : «- Et ta femme, qu'est-ce que nous en faisons ?// - Tu veux parler de Catherine ? » ( $C B: 137)$ Michel omet les liens matrimoniaux et tente de recouvrer un passé anténatal, une sécurité fœtale, qui a un impact sur la topographie du récit : « Un peu avant l'aube, il s'en allait dormir dans la cuisine. » ( $C B: 71)$ Ce lieu marque l'épitomé du féminin selon une géographie genrée de la demeure familiale. Selon cette géographie, par exemple, la cuisine est le lieu du féminin et le salon, celui du 14. Il faut considérer ici l'évolution de Catherine, la femme-objet qui n'avait pas le droit de cuisiner ( $C B: 83$ ) en comparaison avec : «Elle prépara le repas et mit le couvert avec beaucoup de soin. » $(C B: 99)$

15. Un autre exemple explicite sur ce thème : « Je suis tout près de toi, Michel, si près que je t'entends respirer dans mon ventre comme un tout petit enfant que je porterais. » $(C B: 83)$ 
masculin. Il convient de pousser la métaphore et de voir la cuisine comme le ventre de la maison, le siège de la domination maternelle, dans lequel l'enfant reconstitue un cocon-mère. À ne pas dormir dans le lit conjugal, il échappe à son statut de mari au profit de sa propre infantilisation maintenue par les souvenirs de la présence et dominance maternelle dans la cuisine.

\section{Entre maternité et (a)sexualité}

Les conséquences sur la sexualité apparaissent assez clairement en ce sens qu'une telle conception de la relation Catherine-Michel (mère-fils) semble incestueuse. De par la sacralisation ou totémisation de Catherine qui a transformé la consommation de la chair - à travers l'acte sexuel - en une violation, et la recherche constante de Michel d'un substitut maternel, le rapport sexuel ne peut échapper à la transgression et doit donc être évité à tout prix. Si le couple a quand même des rapports sexuels, une analyse freudienne nous permet peut-être de fournir une explication à ces actes. Il semble en effet que la régression à laquelle aspire Michel participe à l'ignition d'un désir physique, qui peut trouver une explication dans le fait que « the vagina is now valued as a place of shelter for the penis; it enters into the heritage of the womb. » (Freud, 1923 : 145) ${ }^{16}$ Alors, poussé une fois encore par un besoin de sécurité et de protection, I'homme-enfant voit en la pénétration une potentielle fuite du monde adulte écrasant. De plus, Michel est replongé dans un stade œdipien qui renforce I'idée d'une attirance sexuelle envers la mère ${ }^{17}$. Les passages traitant des rapports sexuels - c'est-à-dire quand le «ça » prend le dessus - soulignent le fonctionnement psychique de Michel, dans la mesure où le déplaisir semble alors traduire une intervention de son «surmoi ». Trois scènes traitant du rapport physique entre les deux personnages s'avèrent importantes pour comprendre cette problématique :

16. Notre traduction : « le vagin est à présent considéré comme un refuge pour le pénis : il entre dans I'héritage de l'utérus. »

17. Notons que Freud, discutant de l'夭Edipe, remarque que : «the boy regards his mother as his own property ; but he finds one day that she has transferred her love and solicitude to a new arrival » [le garçon perçoit sa mère comme sa propriété ; mais il se rend compte un jour qu'elle a transféré son amour et sa sollicitude sur un autre] (Freud, 1924 : 313). Le terme de propriété convient tout à fait à notre analyse, puisque Catherine est d'abord femme-objet avant d'être l'objet du désir de Michel. En ce qui concerne «l'amour et la sollicitude », dans le cas de ce roman, c'est Michel qui transfère ses sentiments envers sa sœur, mettant ainsi fin à tout rapport éventuel. Catherine devient la mère, dont il se détache comme un enfant post-CEdipe ou un adolescent. Conséquemment, cela explique l'évolution entre : « Je n'ai pas épousé une cuisinière » $(C B: 83)$, et « [i]l ne remarqua pas l'absence de la servante » (CB:99), car l'objet de son désir a dévié. De fait, quand, à sa rencontre avec Catherine, il explique : « pourquoi réveiller ce qui est passé ? Avec vous, je devenais léger comme celui qui n'a jamais eu d'enfance » ( $C B: 50)$, I'on prend conscience que ce passé ne dormait jamais. Nous irons même jusqu'à postuler que l'attachement à ce passé est tellement fort que Michel ne veut pas s'en détacher. En transférant finalement son désir sur la sœur, il parvient à maintenir ce stade infantile. Catherine (et la servante) sont donc des adjuvants qui lui permettent de ne pas avoir à grandir. 
À ce moment il caressait volontiers Catherine, tout contre sa peau, à la limite du linge. [...] Mais bientôt toute chaleur se retirait de Michel. $(C B: 70-71)$

Vers le matin, Catherine était devenue femme. Michel s'écroula à ses côtés comme un noyé et il répétait : «Tu es le diable, Catherine, tu es le diable. » $(C B: 76)$

Catherine, je te parle, tu m'entends, Catherine, ma femme ? [...] Il l'emmena sur le lit et la posséda avec maladresse et fureur. ( $C B: 116)$

L'évolution du désir consiste d'abord en une tentative échouée, pour ensuite donner lieu à I'aversion de Michel pour Catherine, et finalement, la transformation de cette dernière en un simple objet de revanche. Ces épisodes relèvent d'une volonté d'être homme, mais également d'une volonté de retrouver un confort maternel. Dans tous les cas, Michel est condamné puisqu'il est enfermé dans son infantilisme/infantilisation. Il reste aussi que Catherine n'est pas l'objet primaire qui instigue une pulsion physique. Les conséquences surviennent lors de la phase post-coïtale, marquée par l'intrusion du surmoi et la naissance d'un sentiment de culpabilité. Celui-ci prend la forme d'un rejet. «Tu es le diable » est hautement représentatif de la répugnance et du dégoût ressenti par Michel, comme si Catherine était une tentatrice (la mère) ${ }^{18}$. Ce qui pousse Michel à jouir de la chair n'est que l'agentivité de son «ça », qu'il ne peut maîtriser.

\section{Entre fraternisation et abjection}

Finalement, ce rejet dans la sexualité est aggravé lorsque Michel et Lia sont ensemble. Nous sommes alors témoins du processus d'abjection de la femme-mère. En présence des enfants, Catherine est définitivement séquestrée dans le rôle maternel. L'environnement dans lequel elle doit évoluer est dès lors totalement infantilisé. À juste titre, « $[0] n$ aurait dit une salle d'étude austère gardée par le silence » $(C B$ : $105)$ où « le frère établit une sorte de campement baroque auquel il convia sa sœur. » ( $C B$ : 129) Pour mieux comprendre la notion d'abject, tournons-nous vers Kristeva, pour qui il s'agit d'une réaction physique de répulsion comme face à la vue du vomi, ou « la peau à la surface du lait » résultant en « un spasme de la glotte et plus bas encore, de l'estomac ». (Kristeva, 1980 : 10) D'un point de vue psychanalytique, les répercussions identitaires sont majeures (et pertinentes à notre analyse) :

18. La notion de tentatrice doit seulement être perçue en relation avec le diable, celui qui tente à faire le mal. 
Le dégoût alimentaire est peut-être la forme la plus élémentaire et la plus archaïque de l'abjection. [...] De cet élément, signe de leur désir, « je » n'en veux pas, « je » ne veux rien savoir, « je » ne l'assimile pas, « je » l'expulse. Mais puisque cette nourriture n'est pas un « autre » pour «moi » qui ne suis que dans leur désir, je $m^{\prime}$ expulse, je me crache, je $m^{\prime}$ abjecte. (Kristeva, 1980 : 10-11)

Le but est donc de pouvoir se positionner, d'affirmer son identité indépendamment de ses parents. Dans Les chambres de bois, la thématique de la nourriture participe fortement de la distanciation de Catherine. Quand celle-ci revient au fourneau, elle cuisine « des plats nouveaux », mais bientôt « Lia refusa le vin, la viande, le café et tous les condiments » ( $C B: 104)$, et Michel « s'associa au jeûne de Lia et ne voulut plus rien manger de vif et de coloré. » (CB: 105) Ce passage est crucial tant il met en exergue l'unité des enfants et leur besoin de se positionner ( je n'en veux pas ... je l'expulse ») et de rejeter Catherine. Elle est privée de pénétrer dans leur espace, jusque dans la communion alimentaire. Toujours en relation avec la notion d'hospitalité et de nourriture, Kristeva remarque, dans Étrangers à nous-mêmes, que la communion permet à l'étranger d'intégrer un groupe :

La rencontre commence souvent par une fête de la bouche : du pain, du sel et du vin. Un repas, communion nutritive. L'un s'avoue bébé affamé, I'autre accueille l'enfant avide : un instant, ils fusionnent dans le rite de I'hospitalité [...] cosmopolitisme d'un moment, fraternité de convives qui apaisent et oublient leurs différences, le banquet est hors temps. (Kristeva, $1988: 22-23)$

Catherine représente cet étranger en mal d'intégration, dont la nourriture pourrait servir à créer une union. D'abord mère-nourricière avide de nourrir ce « bébé affamé », « [e]lle prépara le repas et mit le couvert avec beaucoup de soin » ( $C B: 99)$, pour se retrouver finalement exclue au plus haut point. Le jeûne des enfants et le rejet, par ces derniers, de la cuisine de Catherine finalisent, de fait, le procédé de distanciation et d'asexuation. Alors que le roman voit le retour du duo Michel-Lia et que Catherine est maternalisée sans possibilité de changement, la sexualité est complètement évacuée dans le couple des mariés. Et sachant que «si quelqu'un personnifie l'abjection, sans promesse de purification, c'est une femme, "toute femme", la "femme toute" » (Kristeva, 1980 : 101-102), Catherine est vouée une fois de plus à un futur de figuration, à rester dans sa chaise haute, témoin du spectacle des enfants, intouchée, intouchable, voire à peine existante à leurs yeux : elle est une femme-souillure ${ }^{19}$.

19. La souillure est ici double, puisqu'il s'agit à la fois d'une référence à la biologie féminine, à savoir la menstruation. Si Catherine devient femme après leur premier rapport, c'est qu'il y a comme un déclenchement de son cycle hormonal et donc d'une «souillure » mensuelle. De même, considérant que nous avons affaire à une construction œdipienne de la relation Catherine-Michel, la souillure résulte de 
Entre émancipation et quête maternelle

Alors, qu'en est-il réellement de l'émancipation de Catherine ? Boisclair conclut que « la présence d'une instance maternelle bienfaisante [...] assure la sauvegarde de sa fille en tentant de l'empêcher d'entrer dans l'économie patriarcale, puis, échouant, se remet à l'ouvrage et réussit à l'en extraire ». (Boisclair, 2005 : 96) Dans une certaine mesure, une telle vision est tout à fait correcte et valide, mais peut-on vraiment dire que la jeune fille est « émancipée [...] de l'économie patriarcale » (Boisclair, 2005 : 82) ? Certes, la rencontre avec Bruno semble l'avoir extraite et sauvée de la relation problématique, voire malsaine, avec Michel, pour lui permettre enfin de faire l'expérience d'une relation plus équilibrée, dans laquelle elle compte comme un individu à part entière. Bruno remarque à propos du mariage : «C'est à vous de décider, Catherine. » ( $C B: 184)$ Par ces mots, l'agentivité est redonnée à la jeune fille qui finit par accepter ${ }^{20}$. Boisclair ajoute que «l'oisiveté n'est guère plus enviable [que la servitude domestique] et que, de toute façon, elle ne garantit pas la non-servitude des rapports sexuels ». (Boisclair, 2005 : 95) Le mariage est donc une des alternatives les plus souhaitables pour l'époque. Si l'on peut voir en cela un certain dilemme cornélien, nous sommes surtout témoins d'un problème sous-jacent plus important : Catherine n'a pas de mère. Par cela, il faut comprendre que le choix final traduit un certain dépit dans la mesure où la réelle recherche de Catherine était celle d'une figure maternelle. Elle reste accrochée à l'idée d'être une jeune fille et d'avoir une mère, de faire partie d'un ensemble féminin. En effet, le processus d'infantilisation affecte aussi I'héroïne, ce qui s'explique donc par une volonté de récréer une relation mère-fille dont elle a été dépourvue. Dans l'optique de Boisclair, Lequin s'intéresse plus précisément à «l'appropriation du corps féminin par la femme » (Lequin, 2009 : 195) et analyse ce fonctionnement chez Hébert : « Pour lutter contre cette négation névrotique, Catherine n'a que le cri et les longs bains sensuels. Intuitivement, elle sait que la proclamation de sa sensualité la sauvera de cette immobilité inhumaine. » (Lequin, 2009 : 199) La référence aux bains et aux cris prend une dimension infantile ${ }^{21}$, et requiert que nous regardions la citation originale en détail : la violation de la chair. Ou, comme dirait Kristeva, « [I]a souillure, c'est l'inceste comme transgression des limites du propre. » (Kristeva, $1980: 101)$

20. De même, c'est l'héroïne qui se montre plus entreprenante avec le jeune homme : « Il ne faut jamais dire adieu, Monsieur. [...] Nous nous reverrons sans doute, puisque vous ne partez que demain. Elle tendit sa main au jeune homme qui la serra brusquement. » (CB:162) Les actions ici sont donc effectuées par Catherine.

21. Le travail critique d'Émond s'intéresse à différents symbolismes, dont celui de l'eau. Il remarque que celle-ci « est omniprésente, s'infiltre partout » (Émond, $1984: 157$ ) et suit même les protagonistes jusqu'à Paris, « comme si Michel transportait avec lui son pays d'enfance. » (Émond, 1984 : 158) Dans le cas de Catherine, le bain vient alors recréer cette enfance perdue. De la maison familiale à la maison 
L'eau chaude, les savons parfumés, la baignoire verte comme un creux de feuillage, les crèmes et les parfums la ravissaient sans fin. Elle passait des heures dans l'eau tiède sous des neiges de savon. Elle essayait de retenir sa respiration le plus longtemps possible sous l'eau, pensant aux pêcheurs d'éponges et aux poissons aveugles. ( $C B: 77$; nous soulignons)

Tandis que le postulat de Lequin est tout à fait valide en tant que réappropriation du féminin, nous proposons également qu'il s'agit de la recréation du ventre maternel ; l'eau tiède prend la place du liquide amniotique et le creux de feuillage sert de nid protecteur tel l'utérus. Dans le même temps, l'image de la jeune fille qui retient sa respiration traduit un retour anténatal où l'enfant dépend de la mère jusque pour son oxygène, mais apporte aussi une dimension ludique. Catherine redevient l'enfant qui joue dans son bain ${ }^{22}$. Nous pouvons voir aussi l'eau comme outil de purification. Catherine, la femme-souillure et souillée, retrouve, l'espace d'un instant, une innocence pré-pubère.

Si I'on accepte l'argument de Boisclair sur la présence de la mère, le présent article souhaite postuler qu'en plus de l'existence de la possibilité d'une « présence bienfaisante », Catherine semble projeter sur le monde extérieur ses désirs maternels. En conséquence, s'il est envisageable que «la mère investit le corps d'Aline » (Boisclair, 2005 : 91), il convient de considérer Aline comme un substitut maternel investi par le désir de Catherine ${ }^{23}$. Une fois libérée de Michel, elle peut enfin tenter d'être soimême et arrêter de jouer des rôles qui I'oppressent.

conjugale, et finalement à la maison au bord de la mer, l'eau reste un élément protecteur, un élément ludique de l'enfance.

22. Dans ce sens, notre interprétation se rapproche de celle d'Émond. Il explique : « Catherine peut se blottir en de telles eaux, dans cette baignoire-coquille, comme l'enfant dans les eaux maternelles. Ce retour à la substance originelle lui permet de remonter le temps jusqu'à sa naissance et par-delà ». (Émond, $1984: 179-180$ )

23. Carlshamre semble sceptique quant à l'interprétation de Boisclair, qu'elle déconstruit dans son travail. Entre autres, elle note : « Il est vrai que c'est la maladie d'Aline qui fait que Bruno reporte son départ, mais il y a un grand pas à franchir pour passer de ce constat à l'idée selon laquelle cette mort serait l'œuvre de la mère, ou même, de manière plus générale, un phénomène positif pour Catherine. Nous considérons au contraire que le contexte montre à quel point Aline correspond à la mère traditionnelle, patriarcale, qui refuse la liberté de sa fille et qui ne vit qu'à travers ses enfants (dans le cas d'Aline, à travers ses maîtres/maîtresses-enfants). » (Carlshamre, 2009 : 79) On peut accepter ces deux visions, il semble en effet qu'Aline ne voit pas d'un bon œil la nouvelle relation de Catherine et Bruno. Toutefois, un peu de scepticisme s'impose aussi quant à considérer Aline comme un personnage traditionnel. Ne pouvons-nous pas voir en sa mort le signe de son opposition au mariage, le symbole de I'oppression ? Notons que le comportement d'Aline a changé. Elle n'était pas très agréable avec Catherine quand celle-ci se laissait diriger par Michel, mais la suit quand elle le quitte comme pour s'assurer qu'elle ne répétera pas le même schéma. 
Entre infantilisation et nouveaux départs

Maintenant qu'elle n'est plus un substitut maternel, elle veut en trouver un pour elle. Aline, la servante, est donc parfaite pour entreprendre cette tâche, car, après tout, une mère n'est-elle pas au service de sa famille?

Souvent, le soir, I'envie d'être bercée prenait Catherine, en son grand lit calme. Elle retenait la servante sous toutes sortes de prétextes : le drap qui pend à gauche, la veilleuse qui flambe comme le jour, le rideau de cretonne qui claque contre la vitre, le verre d'eau qu'on a oublié, le volet qui est mal attaché. Elle aimait suivre les allées et venues de cette grande femme célébrant avec ses mains fortes des offices autour de son lit. $(C B: 150)$

Nous faisons face à une reconstruction d'une routine familiale (I'enfant bercé, mis au lit, bordé...) qui permet à Catherine de recouvrer son enfance perdue. Il est intéressant de voir que de tels moments ont eu lieu dans les deux premières parties, mais l'absence d'un réel substitut maternel pour Catherine coupait court à ses désirs : «toute petite fille » $(C B: 68)$; « petite fille fantasque » $(C B: 79)$; «le corps menu $[\ldots]$ bien dessiné, à peine gonflé aux seins ». ( $C B: 75)$ Autant d'expressions soulignent I'infantilisation de Catherine, qui ne peut cependant pas durer dans la mesure où elle ferait de la concurrence à Michel (et à Lia), alors qu'elle doit les servir comme (une) mère. En ce sens, Catherine ne paraît pas si différente de Michel. Dès lors qu'elle est sous la protection d'Aline, elle adopte un comportement étrangement similaire au sien. Elle dit à Aline : «Quelle vieille sorcière tu fais » $(C B: 165)$, qui résonne avec « $[\mathrm{t}] \mathrm{u}$ es le diable ». $(C B: 76)$ Également, « [e]lle refusa le dîner préparé par Aline » (CB : 157), venant faire écho au jeûne de Michel et de Lia. Ces similitudes font, à nouveau, écho à Kristeva et au besoin d'abjection pour effectuer un positionnement identitaire ; Catherine tente alors de faire face à Aline, la mère-substitut. Bien qu'il y ait là un paradoxe dans le fait qu'elle crée elle-même cette mère et qu'elle veuille s'en détacher, peut-être s'agit-il une fois de plus de la répétition d'un modèle inspiré par Michel ? 
Finalement, la primauté de ce désir d'une relation mère-fille vient également mettre à mal une potentielle sexualité épanouie. La jeune fille ne peut donc pas envisager les rapports sexuels comme le ferait une femme indépendante. Il y a encore une certaine peur face à l'acte; une peur que l'on pourrait justifier par l'absence de mère et l'omniprésence du masculin (un masculin tout aussi asexué : le père veuf et Michel le mari-enfant). La pensée irigarienne nous permet de prendre la mesure de l'importance d'une relation mère-fille positive pour mettre fin à un système principalement phallocentrique. Dans Je, tu, nous, Iragaray fournit des conseils pour développer cette relation et souligne l'importance du rôle de la mère : « I'd also advise them to display photographs of themselves with their daughter(s), or maybe with their mother. [...] The point of these representations is to give girls a valid representation of their genealogy, an essential condition for the constitution of their identity. » (Irigaray, $1990: 41-42)^{24}$ La question de l'héritage, de la descendance et de la généalogie est clairement problématique dans Les chambres de bois, puisque Catherine est dépourvue d'une mère, de modèles maternels, à l'âge même où elle fait face à l'inévitable évolution du corps. Son parcours tout au long du roman consiste à négocier les changements, en favorisant une quête première, à savoir la reconstitution d'une certaine généalogie, pour qu'enfin elle puisse être responsable de son identité.

Le défi initial pour Catherine est donc de se définir en tant qu'individu indépendant, ce qui est vite compromis. Elle tente en vain de former une identité basée sur les souvenirs maternels : «Voici un collier et ce grand châle brodé qui vient de ta mère [...] Elle enfouit sa tête dans le châle qu'elle se mit à mordiller, afin que la senteur de la mère lui entrât dans la bouche ». ( $C B: 55$; nous soulignons) Le legs familial et I'héritage par la fille des attributs féminins de la mère (collier et châle) font partie d'un système de descendance censé aider Catherine à être femme. Malheureusement, telle une plante, la greffe ne prend pas. Aussi fort qu'elle respire et avale «la senteur de la mère », la pénétration échoue, la transformant en un individu autre. C'est pourquoi Michel, «ne paraissant pas reconnaître Catherine [...] répéta : "C'est vous, Catherine ?" ». (CB: 58) Comment peut-elle alors se positionner en tant que femme quand les clés de son agentivité sont dans les mains des autres ? En résulte un inévitable processus qui emprisonne l'héroïne dans une impossibilité de se définir à sa guise. Elle est rapidement objectifiée et maternalisée, perdant ainsi le contrôle de sa construction identitaire et de sa sexualité. Elle, qui voyait le mariage comme une

24. Notre traduction : « Je conseillerais aussi d'avoir des photographies d'elles-mêmes avec leur(s) fille(s), ou bien avec leur mère. [...] Le but de ces représentations est de donner une représentation convenable de leur généalogie, une condition essentielle à la construction de leur identité. » 
issue de secours et la possibilité de vivre avec Michel, prend rapidement conscience de l'influence de la société et des difficultés d'y échapper. Anne Hébert fait donc la lumière sur la servilité de chacun au profit de la continuation d'un modèle social oppressif. Toute performance ayant des fins de conformation et de normalisation ne peut cependant durer, révélant alors des désirs personnels déviants de la norme. Enfin, il serait intéressant de voir comment l'écriture de la nature dans Les chambres de bois sert d'écho à ces problématiques de sexualité et de normalité, mais c'est là un autre projet. 


\section{Bibliographie}

ANOLL, Lidia (1996), "Amors i identidad a l'obra d'Anne Hébert, Les chambres de bois », dans Marta Segarra et Angèls Carabi (dir.), Amor e Identitad, Barcelona (Espagne), Promociones y Publicaciones Universitarias, coll. « Mujeres y Literatura » : 221-231.

AYLWIN, Ulric (1967), "Au pays de la fille maigre : Les chambres de bois d'Anne Hébert », Voix et images du pays, vol. 1, avril : 37-50.

BIRON, Michel, François DUMONT et Élisabeth NARDOUT-LAFARGE (2007), Histoire de la littérature québécoise, Montréal, Boréal.

BISHOP, Neil B. (1977), La thématique de l'enfance dans l'œuvre poétique et romanesque d'Anne Hébert, thèse de doctorat, Université de Provence I.

BOISCLAIR, Isabelle (2000), «Au pays de Catherine », Les Cahiers Anne Hébert, n 2 : 111-125.

BOISCLAIR, Isabelle (2005), « Autonomie et ordre maternel dans Les chambres de bois d'Anne Hébert », Les Cahiers Anne Hébert, nº 6 : 81-89.

BOISCLAIR, Isabelle (2008), " La solidarité féminine comme réponse à la domination masculine : étude de deux motifs genrés dans l'œuvre d'Anne Hébert », Les Cahiers Anne Hébert, nº 8 : 15-36.

BOUCHARD, Denis (1977), Une lecture d'Anne Hébert, Montréal, Hurtubise.

BUTLER, Judith (1997), The Psychic Life of Power : Theories of Subjection, Stanford, Californie, Stanford University Press.

BUTLER, Judith (2004), Undoing Gender, New York, Routledge.

CARLSHAMRE, Katarina (2009), Pulsion et résistance ; émancipation, liberté, et tendances conservatrices dans trois romans d'Anne Hébert, thèse de doctorat, Université de Stockholm.

CHODOROW, Nancy (1978), The Reproduction of Mothering : Psychoanalysis and the Sociology of Gender, Berkeley, University of California Press.

ÉMOND, Maurice (1984), La femme à la fenêtre : l'univers symbolique d'univers d'Anne Hébert dans Les chambres de bois, Kamouraska et Les enfants du Sabbat, Sainte-Foy, Les Presses de l'Université Laval, coll. «Vie des lettres québécoises », n²2.

FREUD, Sigmund (1912), Totem et tabou : Interprétation par la psychanalyse de la vie sociale des peuples primitifs, trad. Samuel Jankélévitch, http://therapiesenligne.be/Freud_Totems_Tabous.pdf.

FREUD, Sigmund (1923), « The Infantile Genital Organization (An Interpolation into the Theory of Sexuality) ", The Standard Edition of the Complete Psychological Works of Sigmund Freud, vol. 19, (19231925). The Ego and the Id and Other Works, London, Vintage.

FREUD, Sigmund (1924), "The Dissolution of the OEdipus Complex », dans On Sexuality, Harmondsworth, Penguin, coll. « The Penguin Freud Library », vol. 7 : 313-322.

GREEN, Mary Jean (2001), Women and Narrative Identity : Rewriting the Quebec National Text, Montréal, McGill-Queen's University Press.

HÉBERT, Anne (1958), Les chambres de bois, Paris, Seuil.

IRIGARAY, Luce (1990), Je, tu, nous, trad. de Alison Martin, New York, Routledge.

JAY, Nancy (1992), Throughout your Generations Forever, Chicago, University of Chicago Press. 
KANT, Emmanuel (1784), « Was it Aufklärung ? », http://www.allmendeberlin.de/What-is-Enlightenment. pdf.

KRISTEVA, Julia (1980), Pouvoirs de l'horreur, Paris, Seuil.

KRISTEVA, Julia (1988), Étrangers à nous-mêmes, Paris, Seuil.

LAPIERRE, Michel (1998), "Catherine : Les chambres de bois ", dans La Vénus québécoise avec ou sans fourrure : la femme dans le roman québécois de 1880 à nos jours, Montréal, Stanké, coll. " L'Isle lettrée »: 117-119.

LEQUIN, Lucille (2009), « La représentation du corps dans la littérature québécoise. De Medjé Vézina à Nelly Arcan », Intercâmbio, $2^{\mathrm{e}}$ série, $\mathrm{n}^{\circ} 2$ : 194-215.

MITCHELL, Juliet (1974), Psychoanalysis and Feminism: A Radical Reassessment of Freudian Psychoanalysis, New York, Basic Books.

RUSSEL, Delbert W. (1983), Anne Hébert, Boston, Twayne Publishers, coll. « Twayne's World Authors Series $", n^{\circ} 684$.

WATTEYNE, Nathalie [et al.] (2008), Anne Hébert : chronologie et bibliographie des livres, parties de livres, articles et autres travaux consacrés à son œuvre, Montréal, Les Presses de l'Université de Montréal, coll. "Espace littéraire ». Cet outil de recherche est mis à jour et disponible sur le site : http://www.usherbrooke.ca/centreanne-hebert/recherche. 